SU-ITP-11/23

SLAC-PUB-14441

\title{
Generalized Attractor Points in Gauged Supergravity
}

\author{
Shamit Kachru, ${ }^{* \dagger}$ Renata Kallosh, ${ }^{*}$ and Marina Shmakova* \\ * Department of Physics and SITP, Stanford University \\ Stanford, CA 94305-4060, USA \\ $\dagger$ Department of Particle Physics and Astrophysics, SLAC \\ Menlo Park, CA 94309, USA

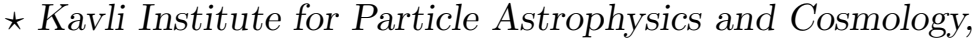 \\ SLAC and Stanford University, Menlo Park, CA 94309, USA \\ kallosh@stanford.edu, skachru@stanford.edu, shmakova@slac.stanford.edu
}

\begin{abstract}
The attractor mechanism governs the near-horizon geometry of extremal black holes in ungauged 4D $\mathrm{N}=2$ supergravity theories and in Calabi-Yau compactifications of string theory. In this paper, we study a natural generalization of this mechanism to solutions of arbitrary 4D $\mathrm{N}=2$ gauged supergravities. We define generalized attractor points as solutions of an ansatz which reduces the Einstein, gauge field, and scalar equations of motion to algebraic equations. The simplest generalized attractor geometries are characterized by non-vanishing constant anholonomy coefficients in an orthonormal frame. Basic examples include Lifshitz and Schrödinger solutions, as well as AdS and dS vacua. There is a generalized attractor potential whose critical points are the attractor points, and its extremization explains the algebraic nature of the equations governing both supersymmetric and non-supersymmetric attractors.
\end{abstract}




\section{Contents}

1 Introduction

2 Generalized Attractors in $\mathrm{D}=4$ Supergravity 5

2.1 Geometry of Supergravity Attractors ............... 7

3 Gauged N=2 D=4 Supergravity $\quad 10$

3.1 Fermionic shifts in generalized attractors of gauged supergravity . . . . . . 12

3.2 Algebraic bosonic equations of motion at the attractor points . . . . . 13

4 Killing Spinors, BPS conditions and Equations of Motion $\quad 15$

4.1 Solutions with unbroken supersymmetry .............. 17

5 Attractor Potential, BPS and Non-BPS Critical Points $\quad 20$

\begin{tabular}{ll|l|}
6 & Examples & 21 \\
\hline
\end{tabular}

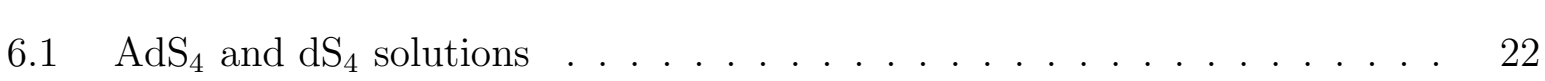

6.2 Lifshitz solutions ...................... . . . 22

6.3 Schrödinger solutions ........................ 24

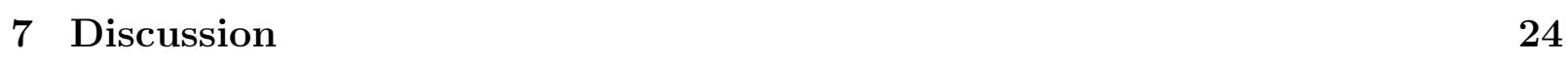

8 Appendix A: Lifshitz geometry $\quad 26$

9 Appendix B: Schrödinger curvatures $\quad 27$ 


\section{Introduction}

The study of the attractor mechanism has been a rich source of insights, both into the physics of black holes [1, 2] and into more formal aspects of string theory [3, 4. In the best understood case of BPS black holes in $4 \mathrm{D} \mathrm{N}=2$ (ungauged) supergravity, the original papers [1, 2] demonstrated that (vector multiplet) scalars are attracted to universal fixed points at the black hole horizon, independent of their asymptotic values at infinity. These fixed points are functions only of the charges of the black hole and the geometry of the moduli space, and can be found by minimizing a suitable attractor potential. We will call the solutions where the scalars take the attractor values even asymptotically, so they remain constant instead of undergoing an attractor flow, and where the gauge fields are also constants when expressed in terms of a suitable tetrad, attractor points. In this language, the attractor points appearing in the original papers [1, 2] are the $A d S_{2} \times S^{2}$ near-horizon geometries of the associated extremal black holes.

Several recent developments have encouraged us to revisit the attractor mechanism and the classification of attractor points in a more general context. On the one hand, there have been serious efforts to generalize the attractor mechanism to $\mathrm{N}=2$ gauged supergravity solutions; see for example the recent work [5, 6] and references therein. We are able to extend these results to provide a simple characterization of the attractor points for general $\mathrm{N}=2$ gauged supergravities.

On the other hand, largely motivated by developments at the interface of AdS/CFT and condensed matter physics [7, there has been intense investigation of solutions with more general asymptotics than Minkowski or AdS. These more general asymptotic metrics can reflect, for instance, the symmetry groups of holographically dual field theories with Galilean [8, 9] or Lifshitz [10] symmetries, which typically arise in condensed matter systems that do not enjoy emergent Lorentz invariance in the infrared. We will find that the emergence of such metrics in $\mathrm{N}=2$ gauged supergravities is closely related to the attractor mechanism, and such metrics characterize generic classes of attractor points with null or timelike Killing vectors. In all of these generalized attractor solutions, the scalar fields take constant values, the gauge fields and Riemann curvature are constants when expressed in terms of a suitable orthonormal tetrad, and the equations of motion simplify from differential equations to algebraic equations.

We explain our strategy below and apply it to the case of $\mathrm{D}=4 \mathrm{~N}=2$ gauged supergravity; similar ideas should of course work for further extended supergravities and other dimen-

sions. As in the past, one may expect that many features of the attractor points we find will 
be universal, and will be present in more general theories with and without supersymmetry.

While to our knowledge the generality of circumstances in which one can find attractor points governed by algebraic equations is a novel result of our work, several recent papers played an important role in motivating us to seek these simplifications. The papers [11, 12], which instigated our work, explored Lifshitz and Schrödinger solutions in particular gauged $\mathrm{N}=2$ supergravities. The earlier papers [13, 14, 15] studied similar solutions from a higher-dimensional perspective; their low-energy effective theories are similar gauged supergravities. The papers [16] investigated the attractor mechanism for a class of nonsupersymmetric black branes, mostly focusing on attractor flows with AdS asymptotics and Lifshitz near-horizon geometries. Our work is a step towards extending the considerations of these papers to find the most general attractors in the context of fully general $\mathrm{N}=2$ gauged supergravities, where (somewhat surprisingly) the problem is tractable. We also find a geometric characterization of the simplest generalized attractors: their geometry has constant anholonomy coefficients, which implies that the tangent space curvature components are constant and non-singular.

The organization of this paper is as follows. In $\S 2$, we define the generalized attractors in gauged supergravity and explain their geometry, in particular, the importance of the tangent space and the concept of anholonomy coefficents. In $\S 3$, we introduce the generic class of 4D $\mathrm{N}=2$ gauged supergravities we will study. We generalize the definition of 'fermionic shifts' to the case of solutions with constant vector fields and fluxes. We show that for attractor points, configurations with constant scalar fields and constant gauge fields in an appropriate sense, the result is a set of algebraic equations which replace the Einstein equations and the other bosonic equations of motion. In $\S 4$ we study supersymmetric attractors. We outline a basic ingredient in our strategy, which is to use Killing spinor identities to help guarantee that certain supersymmetric configurations satisfy all equations of motion. In $\S 5$ we describe a generalized attractor potential whose extrema determines the attractor points. $\S 6$ contains a few simple examples of generalized attractor points, while we close with a discussion in $\S 7$. In Appendix A we present the set of all constant anholonomy coefficients specifying the anisotropic Lifshitz geometry and the set of all constant non-singular tangent space components of the curvature tensor, contrasted with the spacetime dependent curved space components of the curvature tensor. In Appendix $\mathrm{B}$ we present the analogous data for the Schrödinger geometry. 


\section{Generalized Attractors in $\mathrm{D}=4$ Supergravity}

We are interested in finding a generalization of the attractor mechanism of [1, 2] for cases which include not only constant values of the scalars $\phi^{i}$ and tangent space field strengths $F_{a b}=e_{a}^{\mu} e_{b}^{\nu} F_{\mu \nu}$ (fluxes), but also constant values of the tangent space vectors $A_{a}=e_{a}^{\mu} A_{\mu}$. While in the ungauged $\mathrm{N}=2$ supergravity the attractor data depend only on the field strengths $F_{a b}$, in the gauged theory the Lagrangian has additional dependence on $g A_{a}$ (where $g$ is the gauge coupling) that affects the attractor values of the moduli; this explains our interest in solutions with constant $A_{a}$. (Note that, in general, a constant field strength does not require constant vectors).

In the supergravity literature, the attractors are known as critical points of the supergravity action, gauged or ungauged, which solve an algebraic form of the equations of motion - in particular, algebraic BPS conditions. At the attractor points, scalars, fluxes and curvature are constant in the tangent space. Examples include the $A d S_{2} \times S^{2}$ near-horizon geometries of extremal black holes with constant scalars and constant values of $F_{01}$ and $F_{23}$. Our generalized attractors in gauged supergravity have an important non-trivial new ingredient, which enters in the algebraic equations determining the attractor point: constant background vector fields 1

More precisely, the special solutions singled out by [1, 2] are the attractor points where the scalars and fluxes take constant values in the entire solution, determined by the black hole charges and the geometry of the moduli space. We will find that the most elegant generalization to fully generic gauged $\mathrm{D}=4 \mathrm{~N}=2$ supergravities requires that we impose the following conditions: the scalars should be constant, and the gauge fields and field strengths should take constant values in the tangent space, in an orthonormal frame.

A simple new condition (which is not strictly necessary, as we shall see below, but which characterizes the simplest generalized attractors with frozen scalars, gauge fields, and field strengths) is that one should have constant values of the anholonomy coefficients $c_{a b}{ }^{c}$ which are sometimes called Ricci rotation coefficients. See for example [17] for details on supergravity tangent space and vierbein formalism, including the discussion of nonholonomic frames. The relation that follows between constant field strengths $F_{a b}^{\Lambda}$ and

\footnotetext{
${ }^{1}$ It is important in the generic gauged supergravity case to have constant $A_{a}$, because unlike in the case of ungauged attractors, the gauge fields (and not just the field strengths) enter directly into the expression for the attractor potential; see $\S 5$. Therefore, we will take this as part of the definition of a generalized attractor point.
} 
constant Abelian vectors $A_{a}^{\Lambda}$ is

$$
F_{a b}^{\Lambda}=c_{a b}^{c} A_{c}^{\Lambda} .
$$

The easiest way to solve this equation with constant gauge fields and field strengths, is if the anholonomy coefficients themselves are constant. We will see in $\S 3$ that, in fact, to get algebraic equations of motion with a fully generic ansatz for the gauge fields, constant anholonomy will be required. In this case, one can directly prove that the tangent space Riemann-Christoffel curvature is constant as well, and so in the simplest ansatz

$$
F_{a b}^{\Lambda}=c_{a b}{ }^{c} A_{c}^{\Lambda}=\text { const } \quad \Rightarrow \quad \mathrm{R}_{\mathrm{abc}}{ }^{\mathrm{d}}=\mathrm{const}
$$

If in certain directions in the tangent space the fluxes vanish, $F_{\hat{a} \hat{b}}=0$, or the vectors vanish, $A_{\hat{c}}=0$, then the restrictions on the corresponding components of the anholonomy coefficients can be relaxed. It is interesting that spaces with all constant anholonomy coefficients have constant Riemann-Christoffel tangent space curvature which is obviously non-singular. This property is very much in the spirit of the black hole attractors with regular horizon geometry, where scalars reach a particular charge dependent constant value, as opposed to black holes with a singular horizon geometry where scalars have runaway behaviour. The feature of all constant anholonomy coefficients guarantees non-singular constant Riemann-Christoffel curvature in the tangent space 2

Because of the caveat that when certain fluxes or vectors vanish one can relax the corresponding constancy condition on the anholonomy coefficients, constant anholonomy is a sufficient but not a necessary requirement for attractors with constant curvature and fluxes. The earliest examples of attractors in fact exploit this caveat, and do not have constant anholonomy. In particular, in ungauged supergravity, the attractor condition requires only that the non-vanishing

$$
F_{a b}^{\Lambda}=e_{a}^{\mu} e_{b}^{\nu}\left(\partial_{\mu} A_{\nu}^{\Lambda}-\partial_{\nu} A_{\mu}^{\Lambda}\right)
$$

are constant at the attractor point. For example in case of $A d S_{2} \times S^{2}$ attractors in [2] ,

$$
F=\frac{1}{q} d r \wedge d t=\frac{1}{q} e^{0} \wedge e^{1}
$$

and

$$
G=p \sin \theta d \theta \wedge d \phi=p e^{2} \wedge e^{3} .
$$

The tangent space magnetic field in this solution is

$$
A_{3}=e_{3}^{\phi} A_{\phi} \sim \frac{\cos \theta}{\sin \theta}
$$

\footnotetext{
${ }^{2}$ It is known that some of these attractor solutions do have large tidal forces at loci in the space-time; this is true, for instance, of the Lifshitz solutions we discuss in $\S 6$. We have nothing new to say about the proper interpretation of that here.
} 
and the corresponding anholonomy coefficient $c_{23}{ }^{3}$ is not constant - it depends on $\theta$, so that the flux $G_{23}=p$ is constant. This gives an example where the Riemann-Christoffel tangent space curvature $R_{a b c}{ }^{d}$ is constant and non-singular, without fully constant anholonomy. In addition, the gauge field is not constant. However, in the ungauged case, as we mentioned above, the gauge field (as opposed to the field strength) does not enter into the attractor potential, and one need not include constant $A_{a}$ as part of the definition of an attractor. Nevertheless, since our interest is the generalization to generic gauged supergravity, we will for the most part focus on the simplest generalized attractors below. These solutions do exhibit constant anholonomy, in addition to constant vectors, field strengths, and Riemann curvature. Within this class, we will find generic solutions which have emerged as spacetimes of independent interest in the search for gravity duals of non-relativistic field theories.

In what follows, we derive the basic equations governing generalized attractors in gauged supergravity. Because the formalism of $\mathrm{N}=2$ gauged supergravity can obscure the essential points, we give an overview of where we are going in the next subsection. We move on to do the more detailed supergravity analysis in $§ 3$.

\subsection{Geometry of Supergravity Attractors}

Here we briefly summarize some features of the geometry of supergravity. These include concepts like vierbeins, tangent space, orthonormal frame, spin connection, anholonomy coefficients, torsion and curvature tensors; see for example [17] for further details. We will derive here the following statements which are relevant for generalized attractors:

i) in a space-time with constant anholonomy coefficients $c_{a b}^{c}$ (like our simplest generalized attractors), there is a constant non-singular curvature tensor which is determined by the $c_{a b}^{c}$.

ii) the generalized attractor assumption that there are both constant fluxes and constant gauge fields is most simply satisfied with constant anholonomy coefficients, and yields eq. (2.2). More general solutions (as exemplified by the $A d S_{2} \times S^{2}$ solutions above) can also exist, but constant anholonomy is the simplest case (and, as we will see in $\S 3$, the case that guarantees algebraic equations of motion with a fully generic ansatz of constant tangent-space gauge fields and field strengths). 
Given the metric $d s^{2}=g_{\mu \nu}(x) d x^{\mu} d x^{\nu}$, the vierbein $e_{\mu}^{a}$ is related to the metric:

$$
g_{\mu \nu}(x)=e_{\mu}^{a}(x) e_{\nu}^{b}(x) \eta_{a b} \quad a=0,1,2,3
$$

Here $\eta_{a b}$ is the tangent space Minkowski metric. In general relativity, the Lorentz covariant tangent space of supergravity is related to an orthonormal frame. Supergravity fermions live in tangent space: the Dirac equation in curved space is $\gamma^{a} e_{a}^{\mu} D_{\mu} \psi=\gamma^{\mu} D_{\mu} \psi=0$. Here $\gamma^{a}$ is the tangent space numerical Dirac $\gamma$-matrix, $e_{a}^{\mu}(x)$ is an inverse vierbein, and $\gamma^{\mu}(x)=\gamma_{a} e_{a}^{\mu}(x)$. These objects satisfy the relations:

$$
e_{\mu}^{a} e_{b}^{\mu}=\delta_{b}^{a} \quad e_{\mu}^{a} e^{\mu b}=\eta^{a b}
$$

It is convenient to define the vierbein form

$$
e^{a} \equiv e_{\mu}^{a}(x) d x^{\mu} \quad d s^{2}=\eta_{a b} e^{a} e^{b}
$$

and its dual

$$
\tilde{e}_{a} \equiv e_{a}^{\mu} \partial_{\mu} \equiv \partial_{a}
$$

The anholonomy coefficients $c_{a b}{ }^{c}$ are defined via the commutator of the dual vierbein forms $\left[\tilde{e}_{a}^{\mu}(x) \partial_{\mu}, \tilde{e}_{b}^{\nu}(x) \partial_{\nu}\right] \equiv\left[\partial_{a}, \partial_{b}\right] \equiv c_{a b}{ }^{c} e_{c}^{\lambda} \partial_{\lambda}:$

$$
\left[\tilde{e}_{a}, \tilde{e}_{b}\right] \equiv c_{a b}^{c} \tilde{e}_{c}, \quad c_{a b}^{c}=e_{a}^{\mu} e_{b}^{\nu}\left(\partial_{\nu} e_{\mu}^{c}-\partial_{\mu} e_{\nu}^{c}\right)
$$

A Lorentz covariant derivative in the tangent space of supergravity (acting on spinors and tangent space Abelian vectors 3 ) requires the spin connection $\omega$

$$
D_{a}=\partial_{a}+\omega_{a}^{b c} \mathcal{M}_{b c}=e_{a}^{\mu}\left(\partial_{\mu}+\omega_{\mu}^{b c} \mathcal{M}_{b c}\right),
$$

where $\mathcal{M}_{a b}$ is the Lorentz generator. The commutator of two such derivatives, in general, defines the torsion and the curvature tensors of the tangent space:

$$
\left[D_{a}, D_{b}\right]=T_{a b}^{c} D_{c}+R_{a b}^{c d} \mathcal{M}_{c d}
$$

where the tangent space torsion tensor is

$$
T_{a b}{ }^{c}=c_{a b}{ }^{c}+\omega_{a b}^{c}-\omega_{b}^{c} a
$$

and the tangent space curvature is

$$
R_{a b c}{ }^{d}=\partial_{a} \omega_{b c}{ }^{d}-\partial_{b} \omega_{a c}^{d}-\omega_{a c}^{e} \omega_{b e}^{d}+\omega_{b c}{ }^{e} \omega_{a e}^{d}-c_{a b}{ }^{e} \omega_{e c}{ }^{d}
$$

\footnotetext{
${ }^{3}$ In non-Abelian cases, the non-Abelian connection term $e_{a}^{\mu} A_{\mu}^{\Lambda} \mathcal{T}_{\Lambda}$ is present, where $\mathcal{T}_{\Lambda}$ is the non-Abelian gauge symmetry generator.
} 
In the case of a Riemannian spacetime, the torsion is absent and we have a relation between the anholonomy coefficients and the spin connection

$$
T_{a b}{ }^{c}=0 \quad \Rightarrow \quad c_{a b}{ }^{c}=\omega_{a b}^{c}-\omega_{b}^{c} a
$$

The inverse relation defines the spin connection in terms of the anholonomy coefficients

$$
\omega_{a, b c}=\frac{1}{2}\left(c_{a b, c}-c_{a c, b}-c_{b c, a}\right)
$$

It follows that when the anholonomy coefficients are constants.

$$
\partial_{a} c_{b c}{ }^{d}=0
$$

the spin connection and the curvature are constant

$$
R_{a b c}{ }^{d}=-\omega_{a c}{ }^{e} \omega_{b e}{ }^{d}+\omega_{b c}{ }^{e} \omega_{a e}{ }^{d}-c_{a b}{ }^{e} \omega_{e c}{ }^{d} .
$$

When we replace the constant spin connections in (2.19) by their expression in terms of the anholonomy coefficients in (2.17), we find the non-singular curvature tensor as a function of constant anholonomy coefficients

$$
R_{a b c}{ }^{d}=R_{a b c}{ }^{d}\left[c_{e f^{g}}\right]
$$

This proves claim i) in the beginning of this subsection.

Our attractor point condition requires vectors and fluxes to be constant. This is possible only if

$$
A_{a}^{\Lambda}=e_{a}^{\mu} A_{\mu}^{\Lambda}=\mathrm{const} \quad F_{a b}^{\Lambda}=e_{a}^{\mu} e_{b}^{\nu} F_{\mu \nu}^{\lambda}=\mathrm{const}
$$

since

$$
F_{a b}=e_{a}^{\mu} e_{b}^{\nu}\left(\partial_{\mu} e_{\nu}^{c}-\partial_{\nu} e_{\mu}^{c}\right) A_{c}=c_{a b}^{c} A_{c} .
$$

Thus, if both $A_{c}$ and $F_{a b}$ are constant, consistency is most obvious if the anholonomy coefficients $c_{a b}{ }^{c}$ are constant (in the cases that they relate non-vanishing components of $A$ and $F$; as described in $\S 2.1$, there are interesting special examples where some components of the gauge fields vanish, and one does not require constant anholonomy in those directions) 4

This justifies our claim ii) in the beginning of this section.

\footnotetext{
${ }^{4}$ Of course one may also contemplate non-trivial anholonomies with cancellations between different terms in the sum over $c$, but the constant anholonomy attractors are the most elegant. They are also the ones which solve algebraic equations of motion for a generic ansatz of constant tangent space fields.
} 
We therefore define the generalized attractors of $\mathrm{D}=4 \mathrm{~N} \geq 2$ gauged or ungauged supergravity as solutions of equations of motion which are reduced to purely algebraic equations, where the tangent space curvature tensor, scalars, and fluxes are constant. In the particular case of gauged supergravity, when also tangent space vectors are non-vanishing constants and the anholonomy coefficients are constant, we find an interesting set of attractors which we will study below. Such solutions exist both with and without unbroken supersymmetry.

To summarize this section: we have motivated the study of generalized attractors of $\mathrm{N}=2$, $\mathrm{D}=4$ supergravity with the following features:

$$
z^{i}=\mathrm{const}, \quad q^{u}=\mathrm{const}, \quad A_{a}=\mathrm{const}, \quad F_{a b}=\mathrm{const}, \quad c_{a b}{ }^{c}=\mathrm{const}, \quad R_{a b}{ }^{c d}=\mathrm{const}
$$

where $z^{i}$ are scalars from the vector multiplets and $q^{u}$ are the quaternions from the hypermultiplets. In the generic case where there are non-vanishing vectors at the attractor points, the attractor values of the scalars will depend on $A_{a}$ and $F_{a b}$, and on all of the parameters entering in the gauged supergravity 'fermionic shifts' (which we define and study in §3), which include the gauge couplings, the Killing vectors gauging the isometries of the special Kähler and quaternionic Kähler vector multiplet and hypermultiplet moduli spaces, and the momentum maps. We note here that our phrasing of the conditions for an attractor point in (2.23) is not gauge invariant; a gauge transformation can clearly turn constant scalars and vectors into non-constant scalars and vectors. However, it is easy to check that the equations of motion transform in a gauge covariant way, so having found a solution of the type (2.23), a gauge transformation will preserve the fact that it solves the equations of motion, while making the solution look more complicated. In $\S 3.2$, we will further motivate choosing the condition of constant scalars (instead of e.g. covariantly constant scalars) in this definition of generalized attractors.

\section{Gauged $\mathrm{N}=2 \mathrm{D}=4$ Supergravity}

$\mathrm{N}=2, \mathrm{D}=4$ supergravities are of particular interest for several reasons. On the one hand, the constraints which follow from $\mathrm{N}=2$ supersymmetry make such theories, to some extent, solvable. On the other hand, they still exhibit a rich structure of quantum corrections and qualitatively new phenomena compared to their counterparts with further extended supersymmetry. Finally, as the low-energy effective theories arising from Calabi-Yau compactifications of type II string theories, they enjoy a special role in studies of string compactifications and dualities as well. 
The complete Lagrangian for gauged $\mathrm{N}=2$ supergravity [21] in presence of $n_{V}$ Abelian vector multiplets and $n_{H}$ hypermultiplets, with generic gauging of the scalar manifold isometries, is presented in [22]. It was used in recent applications (searching for solutions with unbroken supersymmetry) in [5, 6, 11, 12]. Here, we will use various features of generic BPS solutions that were derived in [5], and apply them to the specific case of attractor points with frozen scalars. We follow the notation of [5].

Gauging modifies the Lagrangian in several ways: it requires the use of gauge-covariant derivatives for the scalars, and also the addition of a scalar potential. The bosonic Lagrangian is

$$
\begin{aligned}
\mathcal{L} & =-\frac{1}{2} R(g)+g_{i \bar{\jmath}} \nabla^{\mu} z^{i} \nabla_{\mu} \bar{z}^{\bar{j}}+h_{u v} \nabla^{\mu} q^{u} \nabla_{\mu} q^{v} \\
& +I_{\Lambda \Sigma} F_{\mu \nu}^{\Lambda} F^{\Sigma \mu \nu}+\frac{1}{2} R_{\Lambda \Sigma} \epsilon^{\mu \nu \rho \sigma} F_{\mu \nu}^{\Lambda} F_{\rho \sigma}^{\Sigma}-g^{2} V(z, \bar{z}, q) .
\end{aligned}
$$

Here $i=1, \ldots, n_{V}, \Lambda=0, \ldots, n_{V}$ and $u=1, \ldots, 4 n_{H}$. The covariant derivatives on scalars are

$$
\nabla_{\mu} z^{i} \equiv \partial_{\mu} z^{i}+g k_{\Lambda}^{i} A_{\mu}^{\Lambda}, \quad \nabla_{\mu} q^{u} \equiv \partial_{\mu} q^{u}+g k_{\Lambda}^{u} A_{\mu}^{\Lambda}
$$

and they define the gauging of isometries of the vector and hypermultiplet scalar manifolds with Killing vectors $k_{\Lambda}^{i}(z)$ and $k_{\Lambda}^{u}(q)$, respectively, and coupling constant $g$. The functions $I_{\Lambda \Sigma}$ and $R_{\Lambda \Sigma}$ are determined by special geometry in terms of the holomorphic prepotential, or in more general cases, in terms of the periods $X^{\Lambda}$ and the dual periods $F_{\Lambda}$; we will not need the explicit formulae here.

The scalar potential depends on the Killing vectors and the corresponding triplet of quaternionic moment maps $P_{\Lambda}^{x}$ :

$$
V(z, \bar{z}, q)=\left(g_{i \bar{\jmath}} k_{\Lambda}^{i} k_{\Sigma}^{\bar{\jmath}}+4 h_{u v} k_{\Lambda}^{u} k_{\Sigma}^{v}\right) \bar{L}^{\Lambda} L^{\Sigma}+\left(g^{i \bar{\jmath}} f_{i}^{\Lambda} \bar{f}_{\bar{\jmath}}^{\Sigma}-3 \bar{L}^{\Lambda} L^{\Sigma}\right) P_{\Lambda}^{x} P_{\Sigma}^{x}
$$

where

$$
L^{\Lambda}=\mathrm{e}^{\mathcal{K}(z, \bar{z}) / 2} X^{\Lambda}(z), \quad f_{i}^{\Lambda}=\mathrm{e}^{\mathcal{K} / 2} D_{i} X^{\Lambda}
$$

and $X^{\Lambda}$ are the holomorphic sections governing the special Kähler geometry. The action is invariant under the following supersymmetry variations (up to higher order terms in fermions):

$$
\begin{aligned}
\delta_{\varepsilon} \lambda^{i A} & =i \nabla_{\mu} z^{i} \gamma^{\mu} \varepsilon^{A}+G_{\mu \nu}^{-i} \gamma^{\mu \nu} \epsilon^{A B} \varepsilon_{B}+i g g^{i \bar{\jmath}} \bar{f}_{\bar{\jmath}}^{\Lambda} P_{\Lambda}^{x} \sigma_{x}^{A B} \varepsilon_{B} \\
\delta_{\varepsilon} \zeta_{\alpha} & =i \mathcal{U}_{u}^{B \beta} \nabla_{\mu} q^{u} \gamma^{\mu} \varepsilon^{A} \epsilon_{A B} \mathbb{C}_{\alpha \beta}+2 g \mathcal{U}_{\alpha u}^{A} \tilde{k}_{\Lambda}^{u} \bar{L}^{\Lambda} \varepsilon_{A}, \\
\delta_{\varepsilon} \psi_{\mu A} & =\nabla_{\mu} \varepsilon_{A}+T_{\mu \nu}^{-} \gamma^{\nu} \epsilon_{A B} \varepsilon^{B}+i g S_{A B} \gamma_{\mu} \varepsilon^{B}
\end{aligned}
$$


where $\lambda^{i A}, \zeta_{\alpha}$ and $\psi_{\mu A}$ are the gauginos, hyperinos and gravitinos respectively, and $A=1,2$. The supercovariant derivative in (3.7) is defined as

$$
\nabla_{\mu} \varepsilon_{A}=\left(\partial_{\mu}-\frac{1}{4} \omega_{\mu}^{a b} \gamma_{a b}\right) \varepsilon_{A}+\frac{i}{2} \mathcal{A}_{\mu} \varepsilon_{A}+\omega_{\mu A}{ }^{B} \varepsilon_{B}
$$

The connections $\mathcal{A}_{\mu}$ and $\omega_{\mu A}{ }^{B}$ are associated to the special Kähler and quaternionic Kähler manifolds, respectively; see [22] for more details. Here the gravitino field strength and mass matrix are

$$
T_{\mu \nu}^{-} \equiv 2 i F_{\mu \nu}^{\Lambda-} I_{\Lambda \Sigma} L^{\Sigma}, \quad S_{A B} \equiv \frac{i}{2}\left(\sigma_{x}\right)_{A B} P_{\Lambda}^{x} L^{\Lambda},
$$

and $F_{\mu \nu}^{\Lambda-}=i \bar{L}^{\Lambda} T_{\mu \nu}^{-}+2 f_{i}^{\Lambda} G_{\mu \nu}^{i-} \quad$ (where the ${ }^{-}$denotes the anti-selfdual part of a two-index tensor).

\subsection{Fermionic shifts in generalized attractors of gauged super- gravity}

In the supergravity literature, 'fermionic shifts' were traditionally associated with the part of the supersymmetry transformations of fermions which is present in vacua with constant scalars. Here we slightly generalize the definition of fermionic shifts, to include the presence of constant (tangent space) vectors and fluxes. Thus we drop from (3.5)-(3.6) terms with derivative of scalars and Lorentz covariant derivative on spinors

$$
\partial_{\mu} z^{i}=0, \quad \partial_{\mu} q^{u}=0
$$

but keep $A_{a}$ and $F_{a b}$. Since these are constant at attractors and always contracted with numerical $\gamma$-matrices in the tangent space, these extra terms in the fermionic shifts are also constants and may be treated on the same footing as terms depending on constant scalars. The remaining terms in (3.5)-(3.6) with account of (3.10) define the fermionic shifts in gauged supergravity attractors.

For the gluinos we have the shift $\tilde{\delta}^{B} \lambda^{i A}$ defined as

$$
\tilde{\delta} \lambda^{i A} \Rightarrow\left(\tilde{\delta}^{B} \lambda^{i A}\right) \varepsilon_{B}=i k_{\Lambda}^{i} A_{a}^{\Lambda} \gamma^{a} \varepsilon^{A}+G_{a b}^{-i} \gamma^{a b} \epsilon^{A B} \varepsilon_{B}+i g g^{i \bar{\jmath}} \bar{f}_{\bar{\jmath}}^{\Lambda} P_{\Lambda}^{x} \sigma_{x}^{A B} \varepsilon_{B} .
$$

For hyperinos we have the shift $\tilde{\delta}^{B} \zeta_{\alpha}$ defined as

$$
\tilde{\delta} \zeta_{\alpha} \Rightarrow\left(\tilde{\delta}^{A} \lambda^{i A}\right) \varepsilon_{A}=i \mathcal{U}_{u}^{B \beta} k_{\Lambda}^{u} A_{a}^{\Lambda} \gamma^{a} \varepsilon^{A} \epsilon_{A B} \mathbb{C}_{\alpha \beta}+2 g \mathcal{U}_{\alpha u}^{A} k_{\Lambda}^{u} \bar{L}^{\Lambda} \varepsilon_{A}
$$

Finally, for the gravitino in the tangent space, $\psi_{a A}=\psi_{\mu A} e_{a}^{\mu}$, we are interested in an integrability condition for the unbroken supersymmetry condition $\delta_{\varepsilon} \psi_{a A}=0$, which we 
present in the form

$$
\delta_{\varepsilon} \psi_{a A}=D_{a} \varepsilon_{A}+\left(\tilde{\delta}^{B} \psi_{a A}\right) \varepsilon_{B} .
$$

Then the fermionic shift $\left(\tilde{\delta}^{B} \psi_{a A}\right)$ is

$$
\tilde{\delta} \psi_{a A}=\left(\tilde{\delta}^{B} \psi_{a A}\right) \varepsilon_{B}=\frac{i}{2} \mathcal{A}_{a} \varepsilon_{A}+\omega_{a A}^{B} \varepsilon_{B}+T_{a b}^{-} \gamma^{b} \epsilon_{A B} \varepsilon^{B}+i g S_{A B} \gamma_{a} \varepsilon^{B}
$$

The connections $\mathcal{A}_{\mu}=e_{\mu}^{a} \mathcal{A}_{a}$ and $\omega_{\mu A}{ }^{B}=e_{\mu}^{a} \omega_{\mu A}{ }^{B}$ are associated to the special Kähler and quaternionic Kähler manifolds [21, 22, 5] and

$$
D_{a} \varepsilon_{A}=\left(\partial_{\mu}-\frac{1}{4} \omega_{\mu}^{a b} \gamma_{a b}\right) \varepsilon_{A}
$$

\subsection{Algebraic bosonic equations of motion at the attractor points}

\section{Vector equations}

The dual field strength $G_{\Lambda \mu \nu}$ is defined as a derivative of the action with respect to $F_{\mu \nu}^{\Lambda}$. In absence of gauging

$$
G_{\Lambda \mu \nu} \equiv R_{\Lambda \Sigma} F_{\mu \nu}^{\Sigma}-\frac{1}{2} I_{\Lambda \Sigma} \epsilon_{\mu \nu \gamma \delta} F^{\Sigma \gamma \delta}
$$

The Maxwell equations and Bianchi identities take a simple form

$$
\epsilon^{\mu \nu \rho \sigma} \partial_{\nu} G_{\Lambda \rho \sigma}=0, \quad \epsilon^{\mu \nu \rho \sigma} \partial_{\nu} F_{\rho \sigma}^{\Lambda}=0
$$

and $\left(F^{\Lambda}, G_{\Lambda}\right)$ transforms as a vector under electric-magnetic duality transformations. Gauging breaks the duality symmetry: the Bianchi identities $\epsilon^{\mu \nu \rho \sigma} \partial_{\nu} F_{\rho \sigma}^{\Lambda}=0$ remain the same, however, the gauge field equations of motion change since the action now has additional dependence on $A_{\mu}^{\Lambda}$, not only on $F_{\mu \nu}^{\Lambda}$ :

$$
\epsilon^{\mu \nu \rho \sigma} \partial_{\nu} G_{\Lambda \rho \sigma}=-g\left(h_{u v} k_{\Lambda}^{u} \nabla^{\mu} q^{v}+g_{i \bar{\jmath}} \frac{1}{2}\left(k_{\Lambda}^{i} \nabla^{\mu} z^{\bar{\jmath}}+h c\right) .\right.
$$

We see now in (3.18) that requiring covariantly constant scalars, instead of constant scalars, would be equivalent to causing the right hand side of this equation to vanish (and hence constitute some loss of generality). Since some of the most interesting solutions which characterize holographic RG fixed points (like Lifshitz and Schrödinger solutions) arise with scalars which are constant (in an appropriate gauge) but not covariantly constant, we have chosen the former condition; we would like to include such basic solutions in our definition of generalized attractors. Of course, there may well also be interesting solutions 
with covariantly constant scalars which are however not constant in any gauge; they do not fall under our current definition of generalized attractors.

At the attractor points (2.23) when the gauge fields are non-vanishing, these equations simplify:

$$
\epsilon^{\mu \nu \rho \sigma} \partial_{\nu} G_{\Lambda \rho \sigma}=-g\left(h_{u v} k_{\Lambda}^{u} A^{\mu \Sigma} k_{\Sigma}^{v}+g_{i \bar{\jmath}} k_{\Lambda}^{i} A^{\mu \Sigma} \bar{k}_{\Sigma}^{\bar{j}}\right)
$$

They can also be given in the form

$$
\epsilon^{\mu \nu \rho \sigma} \partial_{\nu} G_{\Lambda \rho \sigma}=A^{\mu \Sigma} C_{\Sigma \Lambda}
$$

where $C_{\Sigma \Lambda}$ is constant at the attractor point. In the tangent space we find

$$
\epsilon^{a b c d} D_{b} G_{\Lambda c d}=A^{a \Sigma} C_{\Sigma \Lambda}
$$

At the attractor point, $G_{\Lambda c d}$ is constant, related to a constant $F_{a b}$ as follows:

$$
G_{\Lambda a b} \equiv R_{\Lambda \Sigma} F_{a b}^{\Sigma}-\frac{1}{2} I_{\Lambda \Sigma} \epsilon_{a b c d} F^{\Sigma c d}
$$

This means that $\partial_{b} G_{\Lambda c d}$ drops out of the gauge field equation and we are left with an algebraic equation

$$
\partial_{b} G_{\Lambda c d}=0 \quad \Rightarrow \quad \epsilon^{a b c d}\left(D_{b}-e_{b}\right) G_{\Lambda c d}=A^{a \Sigma} C_{\Sigma \Lambda}
$$

In the case of Abelian gauging, the connection in $\left(D_{b}-\partial_{b}\right)$ is just a constant spin connection $\omega_{a}^{b c}$

$$
\epsilon^{a b c d} \omega_{a}^{e}{ }_{c} G_{\Lambda e d}=A^{a \Sigma} C_{\Sigma \Lambda}
$$

The fact that the gauge field equations of motion are algebraic at the attractor point is based on the generic properties of the attractor points defined above, and it is universal.

Notice that above, we assumed that the spin connection is constant, and with a fully generic (constant in tangent space) ansatz for the gauge fields and field strengths, the equation (3.24) is only obviously algebraic in this case. But constancy of the spin connection implies, via (2.16), that the anholonomy coefficients $c_{a b}{ }^{c}$ are constant as well. This justifies our focus on the case of constant anholonomy, despite the fact that famous examples like $A d S_{2} \times S^{2}$ satisfy algebraic equations with slightly different assumptions. It might be interesting to explore a range of other ansatzes for the gauge fields and spin connections which could result in algebraic equations as well.

\section{Einstein equations}


In a background with constant scalars, anholonomy, and curvature that also solves the vector field equations above, one can check that the Einstein equations also reduce to algebraic equations. Namely, in

$$
R_{a b}-\frac{1}{2} \eta_{a b} R=T_{a b}^{\mathrm{attr}}
$$

direct inspection of the supergravity action shows that $T_{a b}^{\mathrm{attr}}$ depends only on the space-time independent solutions for the vectors and scalars, and therefore is constant. The left hand side is constant by the definition of the attractor point. Solving the Einstein equations simply requires an identification of constants which makes (3.25) it consistent.

\section{Scalar equations}

Assuming the vector and Einstein equations can be solved for some constant value of the scalars, the equations of motion for the scalars themselves reduce to the condition that a suitable attractor potential (to be discussed in more detail in §5) be extremized:

$$
\partial_{z^{i}} \mathcal{V}_{\text {attractor }}=\partial_{q^{u}} \mathcal{V}_{\text {attractor }}=0
$$

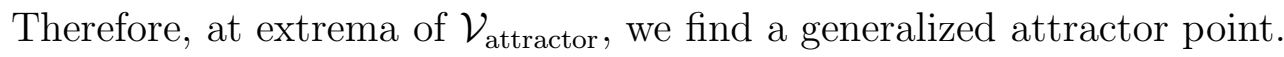

So, we have demonstrated that finding generalized attractor points in gauged $\mathrm{N}=2$ supergravity with $z^{i}, q^{u}, A_{a}^{\Lambda}, F_{a b}^{\Lambda}, c_{a b}^{c}, R_{a b} c d$ all constant, just requires one to solve algebraic equations of motion. However, in general, this is not easy, and unbroken supersymmetry helps. This is the topic of $\S 4$.

\section{Killing Spinors, BPS conditions and Equations of Motion}

In supergravity, a useful shortcut to find particular solutions of the equations of motion, which are second order differential equations, is to solve the conditions for unbroken supersymmetry. For a classical background with vanishing Fermi fields $\psi$, this means one should impose

$$
\delta \psi=0
$$

where $\delta$ is the SUSY variation. These are first order differential equations. They are called BPS conditions because solutions of these equations saturate a BPS bound. Solving these first order differential equations is, in many cases, sufficient or almost sufficient to produce a solution of the full equations of motion, as we now explain. 
Backgrounds which solve (4.1) admit Killing spinors. Using Killing spinor identities [18], one can show that some of the equations of motion are automatically satisfied. The local supersymmetry of the action depending on bosons $\phi^{i}$ and on fermions $\psi^{\alpha}$ means that

$$
\delta_{\varepsilon} S\left(\phi^{i}, \psi^{\alpha}\right)=\frac{\delta S}{\delta \phi^{i}} \delta_{\varepsilon} \phi^{i}+\frac{\delta S}{\delta \psi^{\alpha}} \delta_{\varepsilon} \psi^{\alpha}=0
$$

Here, $\delta_{\varepsilon} \phi^{i}$ is the supersymmetry variation of the bosons and $\delta_{\varepsilon} \psi^{\alpha}$ is the supersymmetry variation of the fermions. Unbroken supersymmetry of a bosonic configuration means that when the fermions vanish, $\psi^{\alpha}=0$,

$$
\delta_{\varepsilon} \psi^{\alpha}=X_{A}^{\alpha}(\phi) \varepsilon^{A}=0
$$

and not all components of $\varepsilon^{A}$ vanish 5 If we differentiate eq. (4.2) over the fermions $\psi^{\beta}$ at $\psi^{\alpha}=0$, then using the facts that $\frac{\delta S}{\delta \phi^{i}}$ and $\delta_{\varepsilon} \psi^{\alpha}$ only have terms which are either independent of the $\psi^{\alpha}$ or of quadratic or higher order in $\psi^{\alpha}$, together with (4.3), we find

$$
\frac{\delta S}{\delta \phi^{i}} \frac{\delta\left(\delta_{\epsilon} \phi^{i}\right)}{\delta \psi^{\beta}}=0
$$

These identities require certain linear combination of the bosonic field equations to vanish. The variational derivative of the supersymmetry transformation of the bosons $\delta_{\varepsilon} \phi^{i}$ with respect to the fermions defines the relevant linear combinations of the equations of motion which are satisfied automatically, when the first order differential equations for unbroken supersymmetry are solved.

Clearly, then, the simplification of the equations of motion in supersymmetric configurations depends both on details of the supersymmetric theory in question and on the number of unbroken supersymmetries. For instance, for minimal 5d supergravity, powerful techniques using Killing spinors were actually used to classify all supersymmetric solutions in [19]. For the particular case of 4D N=2 supergravity, the Killing spinor identities [18, 20] were studed in detail in [20, 5]. It was found there that if one has solved the first order differential equations for unbroken supersymmetry, in a background with a timelike Killing spinor, the Einstein equations and the scalar equations of motion are automatically satisfied. However, one still has to solve equations of motion for the gauge fields 6 . This is of course a considerable simplification. Backgrounds with null Killing spinors are not quite as constrained; for details see [20].

\footnotetext{
${ }^{5}$ Here $A$ runs over the number of supercharges in the N-extended supergravity; for us $N=2$.

${ }^{6}$ When the solution to the equations is given in terms of the gauge field $A_{\mu}$, required in gauged supergravities, the Bianchi identities are automatically satisfied.
} 
In the next subsection, we go through the analysis of the SUSY variations for gauged $\mathrm{N}=2$ supergravities. We will focus on a class of metrics in $\mathrm{D}=4$ spacetime $\left(t, x^{m}\right)(m=1,2,3)$ given by

$$
\mathrm{ds}^{2}=e^{2 U}\left(\mathrm{~d} t^{2}+f_{m} d t d x^{m}\right)-e^{-2 U} \gamma_{m n} \mathrm{~d} x^{m} \mathrm{~d} x^{n}
$$

where $e^{2 U}$ and $f_{m}$ and $\gamma_{m n}$ are independent of $t$. This ansatz is sufficiently general to capture black holes as well as solutions with planar symmetry, like domain walls and plane symmetric solutions of interest in applications of gauge/gravity duality to condensed matter problems. Such metrics often admit Killing vectors (and of course Killing spinors, in the case of BPS solutions).

\subsection{Solutions with unbroken supersymmetry}

For simplicity, we consider here the time-independent static metric in (4.5) with $f_{m}=0$ which corresponds to a frame

$$
e_{t}^{0}=e^{U} \quad e_{x}^{1}=e^{-U} v_{x}^{1} \quad e_{y}^{2}=e^{-U} v_{y}^{2} \quad e_{z}^{3}=e^{-U} v_{z}^{3}
$$

where $\gamma_{m n} d x^{m} d x^{n}=\delta_{i j} v^{i} v^{j}$. We will now discuss how this frame is canonically determined in supersymmetric attractors.

If we have a supersymmetric attractor, then some Killing spinor $\varepsilon_{A}\left(x^{m}\right)$ solves the condition for unbroken supersymmetry arising from the gravitino supersymmetry variation, $\delta_{\varepsilon} \psi_{\mu A}=$ 0 , defined in (3.7). Such spinors anti-commute, but we may also use commuting spinors (by expanding in a basis of Grassmann variables) and relate them to Killing vectors. If by abuse of notation we also call the commuting spinor $\epsilon_{A}$, then we can define

$$
\bar{\epsilon}_{A} \equiv i\left(\epsilon^{A}\right)^{\dagger} \gamma_{0} \quad \text { and } \quad V_{\mu B}^{A} \equiv i \bar{\epsilon}^{A} \gamma_{\mu} \epsilon_{B}
$$

One can then show that this implies that $V_{\mu A}^{A}$ is a timelike or null Killing vector (see [20] for proofs); we will pursue the timelike case below. The dependence of the Killing spinor on $x^{m}$ is

$$
\varepsilon_{A}\left(x^{m}\right)=e^{U} \varepsilon_{A}^{0}
$$

where $\varepsilon_{A}^{0}$ is a constant spinor. Furthermore, we get a tetrad canonically determined by the Killing spinor as follows. We set

$$
V^{\mu} \partial_{\mu}=\sqrt{2} \partial_{t}, \quad \hat{V}^{i}=\frac{1}{\sqrt{2}} V_{J \mu}^{I} \sigma_{I}^{i J} d x^{\mu}
$$


with $\sigma^{i}(i=1,2,3)$ the Pauli matrices. The equations (4.9) define a tetrad canonically determined by the Killing spinor $\epsilon_{A}$; rescaling each by $e^{-U}$ gives rise to an orthonormal tetrad (as in $\S 4$ of the first reference in [20]).

Before proceeding to solve the equations guaranteeing the existence of unbroken supersymmetry, we describe how the unbroken supersymmetry implies the equations of motion. As in [5], denote by $\mathcal{E}_{a}^{\mu}, \mathcal{E}_{\Lambda}^{\mu}, \mathcal{E}_{i}$, and $\mathcal{E}_{u}$ the equations of motion for the vielbein $e_{\mu}^{a}$, the gauge field $A_{\mu}^{\Lambda}$, and the scalars $z^{i}$ and $q^{u}$ (so e.g. $\mathcal{E}_{a}^{\mu}=0$ are the Einstein equations). Then the strategy explained above yields the following Killing spinor identities for supersymmetric backgrounds:

$$
\begin{gathered}
\mathcal{E}_{\Lambda}^{\mu} i f_{i}^{\Lambda} \gamma_{\mu} \epsilon^{A} \epsilon_{A B}+\mathcal{E}_{i} \epsilon_{B}=0 \\
\mathcal{E}_{a}^{\mu}\left(-i \gamma^{a} \epsilon^{A}\right)+\mathcal{E}_{\Lambda}^{\mu}\left(2 \bar{L}^{\Lambda} \epsilon_{B} \epsilon^{A B}\right)=0 \\
\mathcal{E}_{u} \mathcal{U}_{\alpha A}^{u} \epsilon^{A}=0
\end{gathered}
$$

Here, $\mathcal{U}_{u}^{A \alpha}$ is related to the metric on the quaternionic Kähler moduli space by the equation

$$
h_{u v}=\mathcal{U}_{u}^{A \alpha} \mathcal{U}_{v}^{B \beta} C_{\alpha \beta} \epsilon_{A B}
$$

with $C_{\alpha \beta}=-C_{\beta \alpha}$ the flat $S p\left(2 n_{H}\right)$-invariant metric (see $\S 5$ of [22] for further discussion).

One can prove directly from these Killing spinor identities that if the gauge field equations of motion $\mathcal{E}_{\Lambda}^{\mu}=0$ are satisfied, all of the remaining field equations will be satisfied [20. 5].7 So apart from the BPS conditions which we discuss now, to find a supersymmetric attractor point, one needs only to check the gauge field equations of motion (and the Bianchi identities). As we already discussed, for the attractor points, these equations are purely algebraic.

Gravitino. At the attractor point the equation

$$
\delta_{\varepsilon} \psi_{\mu A}=0
$$

simplifies significantly, and so does the integrability condition [17]:

$$
\delta_{\varepsilon} \psi_{\mu A}=0 \quad \Rightarrow\left[D_{a}, D_{b}\right] \varepsilon_{A}=-\frac{1}{4} R_{a b}{ }^{c d} \gamma_{c d} \varepsilon_{A}=-\left(D_{[a} \tilde{\delta} \psi_{b]}+\tilde{\delta} \psi_{a} \tilde{\delta} \psi_{b}\right)_{A B} \varepsilon^{B}
$$

where the derivative $\partial_{a}$ in $D_{a}$ drops, and only the spin connection remains. Here, the fermionic shift is defined in (3.14). $A_{a}, \omega_{a A}^{B}, T_{a b}^{-}$and $S_{A B}$ are constant at the attractor point. They are complicated functions of the vector multiplet scalars $z, \bar{z}$, the hypermultiplet scalars $q^{u}$, and the Killing vectors $k_{\Lambda}^{i}, k_{\Lambda}^{u}$ on the scalar manifolds, and of the momentum

\footnotetext{
${ }^{7}$ This is for a Killing spinor that yields a timelike Killing vector; in the null case, one must also check one of the components of the Einstein equations explicitly [20].
} 
maps $P_{\Lambda}^{x}$ [22]. The integrability condition for the Killing spinors is solved as follows. It reads:

$$
\left(\frac{1}{4} R_{a b}^{c d} \gamma_{c d}-\left(\omega_{[a} \tilde{\delta} \psi_{b]}+\tilde{\delta} \psi_{a} \tilde{\delta} \psi_{b}\right)\right)_{A B} \varepsilon^{B}=0
$$

To solve the algebraic equation (4.16) in a case with maximal unbroken supersymmetry, the terms in brackets must vanish. Otherwise, one finds some constraints on the Killing spinors, so that from the 24 -component spinors $\varepsilon_{A}$, only some fraction may be nonvanishing. (When there are vanishing components of $\epsilon_{A}$, then one should solve the subset of equations following from terms in brackets in (4.16) that multiply the non-vanishing components of the spinor). These equations are algebraic conditions which define the values of $z, \bar{z}, q$ in terms of the other constant parameters in the action and the anholonomy coefficients. We refer to [5] for the details on gauged supergravity integrability equation for gravitino.

The universal feature of the integrability equations for the gravitino is that, at the attractor point, they relate the constant components of the Riemann curvature to the constant values of scalars, vectors and parameters in the action.

Gluino. At the attractor point

$$
\delta_{\varepsilon} \lambda^{i A}=\tilde{\delta} \lambda^{i A B}=0
$$

where the fermionic shift $\tilde{\delta} \lambda$ is defined in (3.11). It is an an algebraic function of the scalars and the parameters appearing in the action and governing the moduli space geometry. As in the gravitino case, one finds some constraints on $\varepsilon_{A}$ and the remaining algebraic equations in (4.17) have to be satisfied.

Hyperino. Here the situation is analogous: at the attractor point

$$
\delta_{\varepsilon} \zeta_{\alpha}=\tilde{\delta} \zeta_{\alpha}=0
$$

where the fermionic shift are given in (3.6) With account of the constraints on $\varepsilon_{A}$, the remaining algebraic equations in (4.18) have to be satisfied. They relate the attractor values of scalars to parameters in the action and the moduli space geometry.

We have seen in this section that to solve all equations of motion in a BPS configuration, one is required only to solve the gauge field equations explicitly; the other equations are then implied by the Killing spinor identities. However, it is often more convenient to find the attractor values of the scalars by minimizing an attractor potential, whose form we now describe. This has the advantage that the extrema of the attractor potential also determine the non-supersymmetric attractor points. 


\section{Attractor Potential, BPS and Non-BPS Critical Points}

In supergravity, generic equations of motion for scalars have terms with two spacetime derivatives, one spacetime derivative, and no spacetime derivatives:

$$
X^{\mu \nu} \partial_{\mu} \partial_{\nu} \phi+X^{\mu} \partial_{\mu} \phi+\frac{\partial \mathcal{V}[\phi, \ldots]}{\partial \phi}=0
$$

Here the potential $\mathcal{V}[\phi, \ldots]$ depends on scalar fields without derivatives, and it may depend on other spacetime dependent fields, like $F_{\mu \nu}$ etc.

The BPS solutions have some unbroken supersymmetry, and they solve scalar equations with at most first derivatives:

$$
Y^{\mu} \partial_{\mu} \phi+\frac{\partial \mathcal{V}_{\mathrm{BPS}}[\phi, \ldots]}{\partial \phi}=0
$$

Here again, $\mathcal{V}_{\mathrm{BPS}}$ depends on scalars without derivatives and on some other spacetime dependent fields.

At attractor points the scalars are constant, so we are looking at the solutions of equations of motion with constant scalars. There is a potential for such supergravity attractors such that the variation of the scalars is an extremum of this attractor potential:

$$
\frac{\partial \mathcal{V}_{\text {attractor }}[\phi]}{\partial \phi}=0
$$

In the case of $D=4 N=2$ gauged supergravity, the attractor potential is given by

$$
\mathcal{V}_{\text {attractor }}(z, \bar{z}, q)=g^{2}\left(V(z, \bar{z}, q)-\left(g_{i j} k_{\Lambda}^{i} k_{\Sigma}^{\bar{j}}+h_{u v} k_{\Lambda}^{u} k_{\Sigma}^{v}\right) A_{a}^{\Lambda} A^{a \Sigma}\right)-\frac{1}{2} \epsilon^{a b c d} F_{a b}^{\Lambda} G_{c d \Sigma} .
$$

Here $V(z, \bar{z}, q)$ is the standard potential given in eq. (3.3.3), and $G_{c d \Sigma}$ also depend on scalars

as shown in eq. (3.22). $F$ and $G$ here are the solutions of the gauge field equations and Bianchi identities where $G$ is a functional of vectors and scalars.

The attractor potential has a beautiful form in terms of the fermionic shifts, defined in Sec. 3. It generalizes the Ward identity to vacua with non-vanishing constant vector potentials and field strengths. Namely as in eq. (9.47) of [22], for N-extended supergravity vacua the scalar potential takes the form:

$$
\delta_{A}^{B} V(z, \bar{z}, q)=Z_{\alpha \beta} \delta_{A} \chi^{\alpha} \delta^{B} \bar{\chi}^{\beta}-3 \mathcal{M}_{A C} \overline{\mathcal{M}}^{C B}
$$

where $Z_{\alpha \beta}$ is the scalar dependent metric on moduli space, $\mathcal{M}_{A C}$ is the scalar dependent gravitino mass matrix, and $\delta_{A} \chi^{\alpha}$ are the fermionic shifts. Here $A=1, \ldots, N$. 
The potential governing our generalized attractor points for general gauged $N=2$ supergravities has a similar structure in terms of fermionic shifts, but the shifts $\tilde{\delta}_{A} \chi^{\alpha}$ and the gravitino mass $\tilde{\mathcal{M}}_{A C}$ include terms depending on constant gauge fields and constant gauge field strengths:

$$
\delta_{A}^{B} \mathcal{V}_{\text {attractor }}(z, \bar{z}, q)=Z_{\alpha \beta} \tilde{\delta}_{A} \chi^{\alpha} \tilde{\delta}^{B} \bar{\chi}^{\beta}-3 \tilde{\mathcal{M}}_{A C} \tilde{\mathcal{M}}^{\mathcal{C B}}
$$

When we differentiate the attractor potential with respect to the scalars, the derivative remains proportional to the fermionic shifts, since the potential is quadratic in them. Thus, $\frac{\partial \mathcal{V}_{\text {attractor }}[\phi]}{\partial \phi}=0$ will be a consequence of the vanishing of the fermionic shifts, and hence of the presence of some unbroken supersymmetry. In such cases, we have supersymmetric attractor points.

Of course, there are also non-supersymmetric attractors; these show up when $\frac{\partial \mathcal{V}_{\text {attractor }}[\phi]}{\partial \phi}=0$ but the fermionic shifts do not vanish. In the case of black holes these have been studied in [2, 23, 24] and many subsequent works.

\section{$6 \quad$ Examples}

In this section, we discuss a few simple examples of our general construction. For general supersymmetric solutions, as discussed in $\S 4$, all equations of motion are guaranteed to be satisfied if we sit at a minimum of the attractor potential and solve the gauge field equations of motion. The novel ingredient is the central role played by metrics of constant anholonomy in the preferred tetrad. Therefore, here we just focus on solving the equations for constant $c_{a b}{ }^{c}$ in an orthonormal tetrad; each such metric should arise in a wide variety of gauged supergravities, and such metrics should be the universal geometries governing attractor points. We will not specify special Kähler and quaternionic Kähler manifolds and particular gaugings which, together with these metrics, give a full solution of supergravity; we expect the metrics we find below to appear in many different specific examples.

We emphasize that all of the geometries we discuss below as examples of constant anholonomy have arisen before in other contexts. Our main purpose here is just to exhibit them emerging, in a unified way, as the simplest and most generic solutions to the equations governing generalized attractor points. 


\section{1 $\quad \mathrm{AdS}_{4}$ and $\mathrm{dS}_{4}$ solutions}

$\mathrm{AdS}_{4}$ solutions of gauged $\mathrm{N}=2$ supergravity are common and well studied. They are also a particular case of the example discussed below in $\S 6.2$ and in Appendix $\mathrm{A}$ : $\mathrm{AdS}_{4}$ is the special case of a Lifshitz geometry with $z=u=1$.

In contrast, dS solutions are not easy to find in gauged supergravities. Generically with non-compact gaugings one can find dS vacua, but typically, there are tachyonic fluctuations in the spectrum and these critical points are unstable. Stable dS vacua in gauged $\mathrm{N}=2$ supergravity were constructed in [25]. The ingredients include non-Abelian non-compact gauging, de Roo-Wagemans rotation angles, and Fayet-Iliopoulos terms.

In terms of geometry, $\mathrm{dS}_{4}$ spaces give one of the simplest examples of constant anholonomy and constant tangent-space curvature. The metric is

$$
d s^{2}=d t^{2}-e^{2 H t} d \vec{x}^{2} .
$$

The constant anholonomy coefficients are (no summation in tangent indices $\hat{k}$ )

$$
c_{0 \hat{k}}^{\hat{k}}=-c_{\hat{k} 0}^{\hat{k}}=-H .
$$

The non-zero Riemann curvature components in the coordinate basis are (no summation on curved space indices $i$ or $j$ )

$$
R_{i, i, t}^{t}=R_{j, j, t}^{i}=-R_{j, i, j}^{i}=-e^{2 H t} H^{2}, \quad R_{t, i, t}^{i}=-H^{2}
$$

Some of them are time-dependent. The tangent space Riemann curvature components are constant. All non-vanishing components of it are

$$
R_{a b c d}= \pm H^{2}
$$

\subsection{Lifshitz solutions}

In light of gauge/gravity duality, in order to find gravity duals of field theories with spatial translation and time translation symmetries, and (in the special case $\gamma_{x x}=\gamma_{y y}$ below) spatial rotation symmetry, it is useful to study the metric ansatz:

$$
d s^{2}=e^{2 U(r)} d t^{2}-e^{-2 U(r)}\left(\gamma_{x x}^{2}(r) d x^{2}+\gamma_{y y}^{2}(r) d y^{2}+d r^{2}\right)
$$

A vielbein and the associated dual tetrad are given by:

$$
e_{t}^{0}=e^{U}, \quad e_{x}^{1}=e^{-U} \gamma_{x x}, \quad e_{y}^{2}=e^{-U} \gamma_{y y}, \quad e_{r}^{3}=e^{-U}
$$




$$
\tilde{e}_{0}=e^{-U} \partial_{t}, \quad \tilde{e}_{1}=\frac{e^{U}}{\gamma_{x x}} \partial_{x}, \quad \tilde{e}_{2}=\frac{e^{U}}{\gamma_{y y}} \partial_{y}, \quad \tilde{e}_{3}=e^{U} \partial_{r} .
$$

The non-trivial anholonomy coefficients are

$$
c_{30}{ }^{0}=-U^{\prime} e^{U}
$$

and

$$
c_{31}{ }^{1}=\left(U^{\prime}-\frac{\gamma_{x x}^{\prime}}{\gamma_{x x}}\right) e^{U}, \quad c_{32}{ }^{2}=\left(U^{\prime}-\frac{\gamma_{y y}^{\prime}}{\gamma_{y y}}\right) e^{U} .
$$

It is easy to find the generic solution with constant anholonomy; the solution of the first equation gives

$$
e^{U}=b_{1} r+b_{2} .
$$

Without loss of generality, we can set $b_{2}=0$ by shifting $r$.

The last two equations $c_{31}{ }^{1}=$ const $\equiv b_{3 x}$ and $c_{32}{ }^{2}=$ const $\equiv b_{3 y}$ now become:

$$
\begin{aligned}
& \frac{\mathrm{d}}{\mathrm{dr}} \log \gamma_{x x}=\left(b_{1}-b_{3 x}\right) e^{-U}, \\
& \frac{\mathrm{d}}{\mathrm{dr}} \log \gamma_{y y}=\left(b_{1}-b_{3 y}\right) e^{-U} .
\end{aligned}
$$

This yields

$$
\begin{aligned}
& \gamma_{x x}(r)=b_{4 x} r^{\frac{\left(b_{1}-b_{3 x}\right)}{b_{1}}} \\
& \gamma_{y y}(r)=b_{4 y} r^{\frac{\left(b_{1}-b_{3 y}\right)}{b_{1}}} .
\end{aligned}
$$

The upshot is that the generic attractor point with planar symmetry is a Lifshitz solution [10] (with, in general, different scaling in the $t, x, y$ directions). The supersymmetry of a class of Lifshitz solutions to some particular $\mathrm{N}=2$ gauged supergravities was established in [11, 12]. Constant scalars, vectors, and field strengths support the solutions in [11, 12], and give nice examples of non-trivial solutions of the algebraic attractor equations of gauged $\mathrm{N}=2$ supergravity described in this paper. The components of the Riemann curvature for the Lifshitz geometry are given in Appendix A; the reader can verify that in the tangent space, $R_{b c d}^{a}$ is constant.

In general, as in [10], we expect that for fully consistent solutions (which do not require e.g. imaginary fluxes to support the background), there will be constraints on the scaling dimensions (e.g. the $z \geq 1$ constraint in [10]). Some familiar solutions arise for particular values of the parameters in the solutions above: $b_{3 x}=b_{3 y}=0$ corresponds to $\mathrm{AdS}_{4}$ solutions, while solutions with $b_{1}=b_{3 x}=b_{3 y}$ correspond to $\mathrm{AdS}_{2} \times \mathrm{R}^{2}$ geometries. That is, these geometries are the extreme limits of Lifshitz solutions where the dynamical critical exponent $z$ goes to 1 or $\infty$. 


\subsection{Schrödinger solutions}

The Schrödinger metric takes the form

$$
d s^{2}=r^{2 z} d u^{2}-2 r^{2} d u d v--\frac{d r^{2}}{r^{2}}-r^{2} d x^{2} .
$$

A convenient vielbein $e_{\mu}^{a} d x^{\mu}$ is given by

$$
e^{0}=\frac{r^{z}}{2} d u, \quad e^{1}=r^{z} d u-2 r^{2-z} d v, \quad e^{2}=r d x, \quad e^{3}=\frac{d r}{r} .
$$

It is a simple matter to compute the $e_{a}^{\nu}=\eta^{a b} e_{\mu}^{b} g^{\mu \nu}$, and from these the anholonomy coefficients. One finds:

$$
c_{30}{ }^{0}=-z, \quad c_{30}{ }^{1}=4(1-z), \quad c_{31}{ }^{1}=2-z, \quad c_{32}{ }^{2}=-1 .
$$

So for all values of $z$, the Schrödinger geometries are also examples of spaces of constant anholonomy, and can be expected to arise at generalized attractor points in various $\mathrm{N}=2$ gauged supergravities. The components of the Riemann curvature tensor for the Schrödinger geometry are given in Appendix B, and are constants in the tangent space basis, as expected. One specific gauged supergravity admitting Schrödinger solutions is discussed in [12]. The paper [15] finds a general correspondence between AdS and Schrödinger solutions; interestingly, their Schrödinger vacua sit at the same attractor values of the moduli as the related AdS vacua.

\section{Discussion}

Many supersymmetric and non-supersymmetric attractor solutions of supergravity are known in $\mathrm{D}=4$. The solutions with constant scalars fit into the general structure of supergravity attractor points described above. An interesting new class of supersymmetric and plane-symmetric solutions with constant scalars and vectors has been identified recently in [11, 12]. These are non-relativistic solutions of $\mathrm{N}=2$ gauged supergravity with constant scalars, including Lifshitz [10], Schrödinger [8, 9], and AdS solutions. The solutions in [11, 12] were based on the study of particular $N=2$ supergravities. They give specific examples of generalized attractors in gauged $\mathrm{N}=2 \mathrm{D}=4$ supergravity. One should expect to find many further examples of interesting attractor points, governed by the algebraic equations discussed here.

We have focused in this paper on the solutions where the scalars are constant over the entire space-time. A more general class of solutions (with or without unbroken supersymmetry) 
interpolates between two different vacua, each of which is characterized by fixed scalars and constant (tangent space) vector fields. The starting and ending points of such interpolating solutions are described by the universal algebraic equations presented in this paper. In all cases, supersymmetric or non-supersymmetric, the scalars at the attractor points are constant, the geometry is characterized by constant anholonomy coefficients, and the equations of motion are algebraic. The extremization of the gauged supergravity attractor potential provides the equations which fix the constant values of scalars. For supersymmetric solutions, one requires the vanishing of the fermionic shifts defined in $\S 3$. More general extrema of the gauged supergravity attractor potential provide non-supersymmetric critical points of gauged supergravity.

It would be interesting to explore analogous simplifications which occur at attractor points in extended supergravities in $\mathrm{D} \neq 4$; to give explicit examples of supersymmetric attractors in $\mathrm{N}=2$ theories with simple canonical choices of the special Kähler and quaternionic Kähler manifold; and, especially, to give a complete classification of the metrics that can possibly arise at attractor points. For instance, under certain assumptions, a classification of nearhorizon geometries of extremal black holes was given in [26]; having such a classification for extremal black brane geometries would be valuable, and would constitute a gauge/gravity duality "prediction" for the possible ground states of holographic matter. In addition, finding in full generality the allowed flows between different kinds of attractor points would be quite interesting, as it would shed light on possible renormalization group flows between dual field theories with different kinds of scale invariance. We leave these problems for the future.

Finally, the attentive reader will have noticed that the reduction of the equations of motion to algebraic equations occurs in this paper due to the simplicity of working with fields that are constant in the tangent space, and is not tied to supersymmetry. For this reason, we suspect it should be possible to find analogous algebraic solutions in many theories even without supersymmetry - in much the same way that the original attractor mechanism for extremal black holes has been generalized to non-supersymmetric theories as well [23, 24].

\section{Acknowledgments}

We are grateful to Sandip Trivedi for numerous stimulating conversations about the attractor mechanism, black hole and black brane solutions, and possible attempts at classifi-

cations thereof. We also thank D. Garfinkle, G. Horowitz, A. Linde, R. Myers, S. Shenker 
and E. Silverstein for enjoyable discussions. We thank M. Mulligan and S. Yaida for helpful comments on a draft. S.K. acknowledges the hospitality of the Aspen Center for Physics while he was thinking about related issues.

\section{Appendix A: Lifshitz geometry}

Here, we work with the anisotropic Lifshitz geometry

$$
d s^{2}=r^{2 z} d t^{2}-r^{2 u} d x^{2}-r^{2} d y^{2}-\frac{d r^{2}}{r^{2}}
$$

(Solutions with different scaling in the $t, x, y$ directions were considered previously in e.g. [27].) The vielbeins are

$$
e_{t}^{0}=r^{z}, \quad e_{x}^{1}=r^{u}, \quad e_{y}^{2}=r, \quad e_{r}^{3}=\frac{1}{r}
$$

The anholonomies are

$$
c_{03}{ }^{0}=z, \quad c_{13}{ }^{1}=u, \quad c_{23}{ }^{2}=1, \quad c_{30}{ }^{0}=-z, \quad c_{31}{ }^{1}=-u, \quad c_{32}^{2}=-1 .
$$

In the coordinate basis $t, x, y, r$, we find the following non-zero components of the Riemann curvature:

$$
\begin{array}{ccc}
R_{x, x, t}^{t}=r^{2 u} u z, & R_{y, y, t}^{t}=r^{2} z, & R_{r, r, t}^{t}=\frac{z^{2}}{r^{2}}, \\
R_{t, x, t}^{x}=r^{2 z} u z, & R_{y, y, x}^{x}=u r^{2}, & R_{r, r, x}^{x}=\frac{u^{2}}{r^{2}}, \\
R_{t, y, t}^{y}=r^{2 z} z, & R_{x, y, x}^{y}=-u r^{2 u}, & R_{r, r, y}^{y}=\frac{1}{r^{2}}, \\
R_{t, r, t}^{r}=r^{2 z} z^{2}, & R_{x, r, x}^{r}=-u^{2} r^{2 u}, & R_{y, r, y}^{r}=-r^{2} .
\end{array}
$$

In the tangent space, with $0,1,2,3$ indices now corresponding to the tetrad in (8.2), we find instead

$$
\begin{array}{ccc}
R_{1,1,0}^{0}=u z, & R_{2,2,0}^{0}=z, & R_{3,3,0}^{0}=z^{2}, \\
R_{0,1,0}^{1}=-u z, \quad & R_{2,2,1}^{1}=-u, \quad R_{3,3,1}^{1}=-u^{2}, \\
R_{0,2,0}^{2}=-z, \quad R_{1,2,1}^{2}=1, & R_{3,3,2}^{2}=-1, \\
R_{0,3,0}^{3}=-z^{2}, \quad R_{1,3,1}^{3}=u^{2}, & R_{2,3,2}^{3}=1 .
\end{array}
$$

Happily, these are constant for all values of $z$ and $u$. 


\section{Appendix B: Schrödinger curvatures}

Here, we work with the Schrödinger spacetime described in $\S 6.3$. The non-zero Riemann curvature components in the coordinate basis $u, v, x, r$ are given by:

$$
\begin{gathered}
R_{u, v, u}^{u}=r^{2}, \quad R_{x, x, u}^{u}=r^{2}, \quad R_{r, r, u}^{u}=\frac{1}{r^{2}}, \\
R_{u, v, u}^{v}=r^{2 z}, \quad R_{v, v, u}^{v}=-r^{2}, \quad R_{x, x, u}^{v}=-r^{2 z}(-1+z), \\
R_{x, x, v}^{v}=r^{2}, R_{r, r, u}^{v}=-2 r^{-4+2 z}(-1+z) z, R_{r, r, v}^{v}=\frac{1}{r^{2}}, \\
R_{u, x, u}^{x}=z r^{2 z}, \quad R_{r, r, x}^{x}=\frac{1}{r^{2}}, \quad R_{u, r, u}^{r}=r^{2 z}(1+(-1+z) z), \\
R_{u, r, v}^{r}=-r^{2}, \quad R_{v, r, u}^{r}=-r^{2}, \quad R_{x, r, x}^{r}=-r^{2} .
\end{gathered}
$$

In the tangent space, with now $0,1,2,3$ indices corresponding to $e_{0}, e_{1}, e_{2}, e_{3}$ in $\S 6.2$, we instead find:

$$
\begin{gathered}
R_{1,0,0}^{0}=-2, \quad R_{1,1,0}^{0}=1, \quad R_{2,2,0}^{0}=4(-1+z), \\
R_{2,2,1}^{1}=1, \quad R_{3,3,0}^{0}=8(-1+z) z, \quad R_{3,3,1}^{0}=1, \\
R_{0,0,0}^{1}=2, \quad R_{0,1,0}^{1}=-1, \quad R_{2,2,0}^{1}=1, \\
R_{3,3,0}^{1}=1, \quad R_{0,2,0}^{2}=4-4 z, \quad R_{0,2,1}^{2}=-1, \\
R_{1,2,0}^{2}=-1, \quad R_{3,3,2}^{2}=-1, \quad R_{0,3,0}^{3}=-8(-1+z) z, \\
R_{0,3,1}^{3}=-1, \quad R_{1,3,0}^{3}=-1, \quad R_{2,3,2}^{3}=1 .
\end{gathered}
$$

We see that happily, the Schrödinger spacetime is characterized by constant Riemann tensor (in the tangent space) for arbitrary values of $z$. 


\section{References}

[1] S. Ferrara, R. Kallosh and A. Strominger, "N=2 extremal black holes," Phys. Rev. D 52, 5412 (1995) arXiv:hep-th/9508072.

[2] S. Ferrara and R. Kallosh, "Supersymmetry and Attractors," Phys. Rev. D 54 (1996) 1514, arXiv:hep-th/9602136;

S. Ferrara, G. W. Gibbons and R. Kallosh, "Black holes and critical points in moduli space," Nucl. Phys. B 500, 75 (1997) [arXiv:hep-th/9702103];

S. Ferrara, K. Hayakawa and A. Marrani, "Lectures on Attractors and Black Holes," Fortsch. Phys. 56, 993 (2008) [arXiv:0805.2498 [hep-th]];

S. Bellucci, S. Ferrara, M. Gunaydin and A. Marrani, "SAM Lectures on Extremal Black Holes in d=4 Extended Supergravity," arXiv:0905.3739 [hep-th];

T. Ortin, "Supersymmetric solutions of 4-dimensional supergravities," AIP Conf. Proc. 1318, 175 (2010) [arXiv:1010.1383 [gr-qc]].

[3] G. W. Moore, "Arithmetic and attractors," arXiv:hep-th/9807087.

[4] H. Ooguri, A. Strominger and C. Vafa, "Black hole attractors and the topological string," Phys. Rev. D 70, 106007 (2004) [arXiv:hep-th/0405146];

J. P. Hsu, A. Maloney and A. Tomasiello, "Black hole attractors and pure spinors," JHEP 0609, 048 (2006) [arXiv:hep-th/0602142].

[5] K. Hristov, H. Looyestijn and S. Vandoren, "BPS black holes in N=2 D=4 gauged supergravities," JHEP 1008, 103 (2010) [arXiv:1005.3650 [hep-th]];

K. Hristov, H. Looyestijn and S. Vandoren, "Maximally supersymmetric solutions of $\mathrm{D}=4 \mathrm{~N}=2$ gauged supergravity," JHEP 0911, 115 (2009) arXiv:0909.1743 [hep-th].

[6] M. Huebscher, P. Meessen, T. Ortin, S. Vaula, "Supersymmetric N=2 EinsteinYang-Mills monopoles and covariant attractors," Phys. Rev. D78, 065031 (2008) [arXiv:0712.1530 [hep-th]];

S. Bellucci, S. Ferrara, A. Marrani, A. Yeranyan, "d=4 Black Hole Attractors in N=2 Supergravity with Fayet-Iliopoulos Terms," Phys. Rev. D77, 085027 (2008). [arXiv:0802.0141 [hep-th]];

M. Huebscher, P. Meessen, T. Ortin, S. Vaula, "N=2 Einstein-Yang-Mills's BPS solutions," JHEP 0809, 099 (2008) [arXiv:0806.1477 [hep-th]];

D. Cassani, S. Ferrara, A. Marrani, J. F. Morales and H. Samtleben, "A Special road to AdS vacua," JHEP 1002, 027 (2010) [arXiv:0911.2708 [hep-th]];

S. L. Cacciatori, D. Klemm, "Supersymmetric AdS(4) black holes and attractors," JHEP 1001, 085 (2010). [arXiv:0911.4926 [hep-th]]; 
G. Dall'Agata and A. Gnecchi, "Flow equations and attractors for black holes in N = 2 U(1) gauged supergravity," JHEP 1103, 037 (2011) [arXiv:1012.3756 [hep-th]];

K. Hristov, S. Vandoren, "Static supersymmetric black holes in $A d S_{4}$ with spherical symmetry," JHEP 1104, 047 (2011). [arXiv:1012.4314 [hep-th]].

[7] S. A. Hartnoll, "Lectures on holographic methods for condensed matter physics," Class. Quant. Grav. 26 (2009) 224002 arXiv:0903.3246 [hep-th];

C. P. Herzog, "Lectures on Holographic Superfluidity and Superconductivity," J. Phys. A A42, 343001 (2009) [arXiv:0904.1975 [hep-th]];

J. McGreevy, "Holographic duality with a view toward many-body physics," Adv. High Energy Phys. 2010, 723105 (2010) [arXiv:0909.0518 [hep-th]];

G. Horowitz, "Introduction to Holographic Superconductors," arXiv:1002.1722 [hepth];

S. Sachdev, "Condensed matter and AdS/CFT," arXiv:1002.2947 [hep-th].

[8] D. T. Son, "Toward an AdS/cold atoms correspondence: A Geometric realization of the Schrodinger symmetry," Phys. Rev. D 78, 046003 (2008) [arXiv:0804.3972 [hepth]].

[9] K. Balasubramanian and J. McGreevy, "Gravity duals for non-relativistic CFTs," Phys. Rev. Lett. 101, 061601 (2008) [arXiv:0804.4053 [hep-th]].

[10] S. Kachru, X. Liu and M. Mulligan, "Gravity Duals of Lifshitz-like Fixed Points," Phys. Rev. D 78, 106005 (2008) [arXiv:0808.1725 [hep-th]].

[11] D. Cassani and A. F. Faedo, "Constructing Lifshitz solutions from AdS," arXiv:1102.5344 [hep-th].

[12] N. Halmagyi, M. Petrini and A. Zaffaroni, "Non-Relativistic Solutions of N=2 Gauged Supergravity," arXiv:1102.5740 [hep-th].

[13] C. P. Herzog, M. Rangamani, S. F. Ross, "Heating up Galilean holography," JHEP 0811, 080 (2008). [arXiv:0807.1099 [hep-th]];

J. Maldacena, D. Martelli, Y. Tachikawa, "Comments on string theory backgrounds with non-relativistic conformal symmetry," JHEP 0810, 072 (2008). [arXiv:0807.1100 [hep-th]];

A. Adams, K. Balasubramanian, J. McGreevy, "Hot Spacetimes for Cold Atoms," JHEP 0811, 059 (2008). [arXiv:0807.1111 [hep-th]].

[14] K. Balasubramanian and K. Narayan, "Lifshitz spacetimes from AdS null and cosmological solutions," JHEP 1008, 014 (2010) [arXiv:1005.3291 [hep-th]]; 
A. Donos and J. P. Gauntlett, "Lifshitz Solutions of D=10 and D=11 supergravity," JHEP 1012, 002 (2010) [arXiv:1008.2062 [hep-th]];

R. Gregory, S. L. Parameswaran, G. Tasinato and I. Zavala, "Lifshitz solutions in supergravity and string theory," JHEP 1012, 047 (2010) [arXiv:1009.3445 [hep-th]];

A. Donos, J. P. Gauntlett, N. Kim and O. Varela, "Wrapped M5-branes, consistent truncations and AdS/CMT," JHEP 1012, 003 (2010) [arXiv:1009.3805 [hep-th]].

[15] P. Kraus, E. Perlmutter, "Universality and exactness of Schrodinger geometries in string and M-theory," [arXiv:1102.1727 [hep-th]].

[16] K. Goldstein, S. Kachru, S. Prakash and S. P. Trivedi, "Holography of Charged Dilaton Black Holes," JHEP 1008, 078 (2010) [arXiv:0911.3586 [hep-th]];

K. Goldstein, N. Iizuka, S. Kachru, S. Prakash, S. P. Trivedi and A. Westphal, "Holography of Dyonic Dilaton Black Branes," JHEP 1010, 027 (2010) [arXiv:1007.2490 [hep-th]].

[17] T. Ortin, "Gravity and strings," Cambridge Unversity, Cambridge University Press, 2004;

S. J. Gates, M. T. Grisaru, M. Rocek and W. Siegel, "Superspace Or One Thousand and One Lessons in Supersymmetry," Front. Phys. 58, 1 (1983) [arXiv:hepth/0108200].

[18] R. Kallosh and T. Ortin, "Killing Spinor Identities," arXiv:hep-th/9306085.

[19] J. P. Gauntlett and J. B. Gutowski, "All supersymmetric solutions of minimal gauged supergravity in five-dimensions," Phys. Rev. D 68, 105009 (2003) [Erratum-ibid. D 70, 089901 (2004)] [arXiv:hep-th/0304064].

[20] P. Meessen and T. Ortín, "The supersymmetric configurations of $\mathrm{N}=2, \mathrm{~d}=4$ supergravity coupled to vector supermultiplets," Nucl. Phys. B 749 (2006) 291, arXiv:hepth/0603099;

M. Huebscher, P. Meessen and T. Ortín, "Supersymmetric solutions of N=2 d=4 sugra: the whole ungauged shebang," Nucl. Phys. B 759 (2006) 228, arXiv:hep-th/0606281.

[21] B. de Wit, A. Van Proeyen, "Potentials and Symmetries of General Gauged N=2 Supergravity: Yang-Mills Models," Nucl. Phys. B 245 (1984) 89;

B. de Wit, P. G. Lauwers, R. Philippe, S. Q. Su and A. Van Proeyen, "Gauge And Matter Fields Coupled To N=2 Supergravity," Phys. Lett. B 134 (1984) 37;

J. P. Derendinger, S. Ferrara, A. Masiero and A. Van Proeyen, "Yang-Mills Theories Coupled To $\mathrm{N}=2$ Supergravity: Higgs And Superhiggs Effects In Anti-De Sitter 
Space," Phys. Lett. B 136 (1984) 354;

B. de Wit, P. G. Lauwers and A. Van Proeyen, "Lagrangians Of N=2 Supergravity Matter Systems," Nucl. Phys. B 255, (1985) 569;

R. D'Auria, S. Ferrara and P. Frè, "Special and Quaternionic Isometries: General Couplings in N=2 Supergravity and the Scalar Potential," Nucl. Phys. B 359, (1991) 705.

[22] L. Adrianopoli, M. Bertolini, A. Ceresole, R. D'Auria, S. Ferrara, P. Fre and T. Magri, "N=2 Supergravity and N=2 Super Yang-Mills Theory on General Scalar Manifolds," J. Geom. Phys. 23, (1997) 111 arXiv:hep-th/9605032.

[23] K. Goldstein, N. Iizuka, R. P. Jena and S. P. Trivedi, "Non-supersymmetric attractors," Phys. Rev. D 72, 124021 (2005) [arXiv:hep-th/0507096].

[24] A. Ceresole, G. Dall'Agata, "Flow Equations for Non-BPS Extremal Black Holes," JHEP 0703, 110 (2007). [hep-th/0702088].

[25] P. Fre, M. Trigiante and A. Van Proeyen, "Stable de Sitter vacua from N=2 supergravity," Class. Quant. Grav. 19, 4167 (2002) [arXiv:hep-th/0205119].

[26] H. K. Kunduri, J. Lucietti, H. S. Reall, "Near-horizon symmetries of extremal black holes," Class. Quant. Grav. 24, 4169-4190 (2007). [arXiv:0705.4214 [hep-th]].

[27] S. S. Pal, "Anisotropic gravity solutions in AdS/CMT," [arXiv:0901.0599 [hep-th]]. 